\title{
Mortalidade materna e qualidade do preenchimento das declarações de óbito em um hospital escola de referência do Ceará
}

\section{Maternal mortality and quality of the filling of death certificates in a Ceará reference school hospital}

Priscila Fiusa Lyra Miná1. Jordana Parente Paiva ${ }^{2}$. Francisco Edson de Lucena Feitosa ${ }^{3}$. Denise Ellen Francelino Cordeiro ${ }^{1}$.

1 Residência em Ginecologia e Obstetrícia, Maternidade Escola Assis Chateaubriand (MEAC), Universidade Federal do Ceará (UFC), Fortaleza, Ceará, Brasil. 2 Mestrado em Ciências Clínico-Cirúrgicas, Universidade Federal do Ceará (UFC). Presidente da Comissão Hospitalar de Prevenção ao óbito materno, infantil e fetal, Maternidade Escola Assis Chateaubriand (MEAC), Fortaleza, Ceará, Brasil. 3 Doutorado em Tocoginecologia, Universidade Estadual de Campinas (UNICAMP). Professor adjunto, Departamento de Saúde Materno-Infantil, Universidade Federal do Ceará (UFC), Chefe da Divisão de Cuidado da Maternidade Escola Assis Chateaubriand (MEAC), Fortaleza, Ceará, Brasil.

\section{RESUMO}

Objetivos: descrever a frequência da mortalidade materna na Maternidade Escola Assis Chateaubriand no período de janeiro de 2011 a julho de 2015 e avaliar o correto preenchimento das respectivas declarações de óbito (DO). Metodologia: estudo descritivo, de coorte, realizado através da coleta de dados de prontuários e das declarações de óbito emitidas no período do estudo. Resultados: sessenta óbitos ocorreram no período do estudo, sendo três excluídos da análise. Dos óbitos, $66,67 \%$ foram classificados como de causa obstétrica direta, 26,32\% obstétrica indireta e 5,26\% não obstétrico. As causas básicas dos óbitos foram distribuídas nos seguintes grupos: hipertensiva 22,81\%; hemorrágica 21,05\%; infecciosa 17,54\%; complicação cirúrgica 7,02\%; tromboembolismo pulmonar 7,02\%; neoplásica 5,26\%; outro 17,54\%. De todos os óbitos, 71,7\% ocorreram no período do puerpério. Foram encontrados $66,67 \%$ de preenchimentos incorretos na causa básica da morte das declarações de óbito emitidas. Dentre os motivos para o preenchimento incorreto, considerou-se o uso de termos vagos (falência ou disfunção de múltiplos órgãos; siglas como AVC) ou uso de termos não classificados na CID-10. Conclusão: sugere-se uma melhor capacitação do profissional médico, uma vez que a DO é um instrumento de preenchimento obrigatório pelo médico, além de principal fonte de informações sobre o perfil de mortalidade do país.

Palavras-chave: Mortalidade materna. Atestado de óbito. Causas de morte.

\section{ABSTRACT}

Objectives: Describe the frequency of maternal mortality in the Maternidade Escola Assis Chateaubriand from January 2011 to July 2015 and evaluate the correct filling of the respective death certificates. Methods: A descriptive, cohort study, accomplished by collecting data from medical records and death certificates issued during the study period. Results: Sixty deaths occurred in the study period, three of which were excluded from the analysis. $66.67 \%$ of the deaths were classified as having a direct obstetric cause, $26.32 \%$ indirect obstetric cause and $5.26 \%$ non-obstetric. The basic causes of deaths were distributed in the following groups: hypertensive $22.81 \%$; hemorrhagic $21.05 \%$; infectious $17.54 \%$; surgical complication $7.02 \%$; pulmonary thromboembolism $7.02 \%$; neoplasic $5.26 \%$; other $17.54 \% .71 .7 \%$ of all deaths occurred in the puerperium. We found $66.67 \%$ of incorrect filling in the basic cause of death of the death certificates issued. Among the reasons for incorrect filling, we considered the use of vague terms (such as dysfunction of multiple organs or acronyms) or use of terms not classified in the ICD-10. Conclusions: A better qualification of the medical professional is suggested, since the death certificate is a compulsory instrument filled by the physician, as well as the main source of information on the country's mortality profile.

Keywords: Maternal mortality. Death certificates. Cause of death.

Autor correspondente: Priscila Fiusa Lyra Miná, Avenida Luciano Carneiro, 635, Fátima, Fortaleza, Ceará. CEP: $60411-205$. Telefone: +55 85 99915-3005. E-mail: priscilafiusa@gmail.com

Conflito de interesses: Não há qualquer conflito de interesses por parte de qualquer um dos autores.

Recebido em: 19 Mar 2018; Revisado em: 16 Mai 2018; Aceito em: 16 Mai 2018. 


\section{INTRODUÇÃO}

A mortalidade materna é um dos índices mais alarmantes de saúde nos países em desenvolvimento devido à evitabilidade em $92 \%$ dos casos. ${ }^{1}$ Estima-se que em 2010 houve 287.000 mortes maternas no mundo, ${ }^{2}$ sendo destas, 1447 no Brasil. ${ }^{3}$

Há muito tempo a redução da mortalidade materna é vista como prioridade na saúde global. Na Cúpula do Milênio da Organização das Nações Unidas (ONU), em 2000, foram adotadas as Metas de Desenvolvimento, entre elas, reduzir a mortalidade materna em 75\% até 2015. ${ }^{4}$ Segundo dados da Organização Mundial da Saúde (OMS), o Brasil reduziu a taxa de mortalidade materna em 43\% de 1990 até 2013. ${ }^{5}$ No entanto, apesar do avanço, o país continua longe de alcançar a taxa objetivada.

No Ceará, de 1998 a 2014, foram notificados 38.120 óbitos de mulheres em idade fértil (MIF), e destes, foram confirmadas 2.039 mortes maternas, sendo 1.802 por causas obstétricas diretas ou indiretas, com uma média da razão da mortalidade materna (RMM), no período supracitado, de 78,1 mortes maternas por 100.000 nascidos vivos, ${ }^{6}$ índice considerado alto segundo parâmetros da OMS.

Um requisito fundamental para maiores avanços na redução das mortes maternas é conhecer o perfil epidemiológico dos óbitos ${ }^{2}$ ocorridos em nosso meio. Pois dessa forma é possível avaliar as deficiências que levam aos maus resultados obstétricos e entender melhor as necessidades do nosso sistema de saúde para que sejam estabelecidas mudanças positivas.

A principal fonte de dados sobre mortalidade no Brasil é o Sistema de Informação sobre Mortalidade (SIM). Por meio desse sistema são desenvolvidas estatísticas relacionadas a estudos epidemiológicos e demográficos que permitem gestão de políticas e ações em saúde. A dificuldade que mais se destaca na construção das estatísticas de mortalidade confiáveis é o adequado preenchimento da declaração de óbito (DO), instrumento de alimentação de dados para o SIM. ${ }^{7}$

A DO tem dois objetivos principais: o primeiro é o de ser o documento padrão para a coleta das informações sobre mortalidade, que servem de base para o cálculo das estatísticas vitais e epidemiológicas do Brasil; o segundo, de caráter jurídico, indispensável para as formalidades legais do sepultamento. Para o cumprimento desses objetivos, é fundamental o empenho e o compromisso do médico com relação à veracidade, completude e fidedignidade das informações registradas na DO, uma vez que é o profissional responsável pelas informações contidas no documento. ${ }^{8}$

Com o intuito de conhecer ou de, ao menos, se aproximar mais do número real de mortes maternas, a seguinte proposta foi feita pela OMS: "Com vistas a melhorar a qualidade dos dados de mortalidade materna e fornecer métodos alternativos de coleta das mortes durante a gravidez ou relacionadas com ela, assim como a encorajar o registro das mortes por causas obstétricas ocorrendo no período além dos 42 dias após o término da gravidez, a Quadragésima Terceira Assembleia Mundial de Saúde adotou, em 1990, a recomendação de que os países considerassem a inclusão, nos atestados de óbito, de questões que dissessem respeito à gravidez atual e à gravidez durante $\mathrm{o}$ ano que precedeu a morte". No Brasil, o Ministério da Saúde, gestor do Sistema de Informação de Mortalidade, passou desde 1995, a considerar essa variável na declaração de óbito (DO). ${ }^{9}$

Em relação especificamente à mortalidade materna, observase que a qualidade no preenchimento das variáveis "óbito na gravidez" e "óbito no puerpério" na DO tem aumentado ao longo dos anos, em torno de $40 \%$ entre 2000 a 2005 e $75 \%$ a partir de 2006, entretanto, ainda são observadas diversas inconsistências em seu preenchimento, apesar dos esforços para melhorar as estatísticas de morte materna. ${ }^{7}$

Tendo em vista que a DO constitui o instrumento padronizado pelo Ministério da Saúde desde 1976 para a coleta de dados de mortalidade no Brasil, é importante a realização de estudos que avaliem seu correto preenchimento e as consequências dessas inconsistências nas informações sobre mortalidade no país.

Desta forma, propusemos este estudo, cujo objetivo é levantar dados relativos às mortes maternas ocorridas na Maternidade Escola Assis Chateaubriand (MEAC) no período de janeiro de 2011 a julho de 2015 e investigar a qualidade do preenchimento das respectivas declarações de óbito emitidas.

\section{METODOLOGIA}

Estudo descritivo, de coorte, com o objetivo de analisar todos os casos de mortes maternas ocorridas na Maternidade Escola Assis Chateaubriand (MEAC) no período de janeiro de 2011 a julho de 2015, além do adequado preenchimento das declarações de óbito. A pesquisa foi aprovada pelo Comitê de Ética da Maternidade Escola Assis Chateaubriand vinculada à Universidade Federal do Ceará (UFC) através do parecer de número 1.421.968. Após aprovação no comitê de ética foram colhidos dados a partir dos prontuários e das declarações de óbito emitidas no período do estudo.

Foram avaliadas as causas básicas do óbito materno e agrupadas em grandes grupos (hipertensiva, hemorrágica, infecciosa, neoplásica, complicação cirúrgica, tromboembolismo pulmonar, outra e ignorada); classificado o óbito quanto ao tipo de morte materna (obstétrica direta, indireta, não obstétrica e ignorada); classificado o período em que ocorreu o óbito (na gestação, no parto, no puerpério, de 43 dias até 1 ano após o parto, durante aborto, após aborto e ignorado); se exame de necropsia foi realizado; qual a data do óbito; qual o responsável pelo preenchimento da DO (médico plantonista da Unidade de Terapia Intensiva (UTI) materna, médico obstetra, médico residente ou outro).

Em relação às declarações de óbito, foram analisados os campos de preenchimento obrigatório, ou seja, do bloco II ao bloco VII, seguindo os seguintes critérios:

(1) não preenchimento ("campos em branco");

(2) preenchimento incompleto; 
(3) campos ilegíveis;

(4) preenchimento incorreto (equivocado);

(5) preenchimento adequado.

Foi utilizado como critério de inclusão as mortes ocorridas em pacientes que se encontravam gestantes, ou ocorridas no período de até um ano após o parto na Maternidade Escola Assis Chateaubriand, no período compreendido de janeiro de 2011 a julho de 2015.

Durante o período do estudo foram catalogados 60 óbitos na MEAC. Desses, três pacientes foram excluídas por: óbito ocorrido fora do período gravídico-puerperal; não ser encontrado o prontuário; óbito ter ocorrido no Hospital Universitário Walter Cantídio e não termos disponível a DO em nosso prontuário. Como instrumento de coleta de dados, foi criada uma ficha pré-codificada para a inserção dos dados, de onde os mesmos foram transcritos pelos investigadores para um banco de dados no programa Microsoft Access.

Os dados foram analisados no programa Microsoft Excel. As variáveis qualitativas foram representadas por frequência absoluta(n) e relativa (\%).

\section{RESULTADOS}

Foram avaliados 57 óbitos e 45 declarações de óbito emitidas no hospital no período do estudo. Os 12 óbitos sem DO, correspondem aos óbitos encaminhados para necropsia. Entre os tipos de morte materna, 66,67\% dos óbitos (38 casos) foram classificados como de causa obstétrica direta; $26,32 \%$ (15 casos) como de causa obstétrica indireta; 5,26\% (3 casos) como de causa não obstétrica (morte encefálica por trauma cranioencefálico (TCE), apendicite aguda, meningoencefalite herpética com morte encefálica no $5^{\circ}$ mês pós-parto); $1,75 \%$ (1 caso) como causa ignorada devido a DO ter sido preenchida com causa de óbito indeterminada.

As principais causas básicas de óbito foram as hipertensivas com 13 casos $(22,81 \%)$ e hemorrágicas com 12 casos $(21,05 \%)$. As demais causas básicas de óbito com seu valor percentual podem ser observadas na Tabela 1. Dentre as causas básicas de óbito classificadas como "outros", destacam-se: hipertensão pulmonar severa prévia à gestação (3 casos) e cardiopatia préexistente (2 casos). Os demais casos, morte encefálica por TCE devido a acidente de moto, insuficiência hepática aguda de causa não esclarecida, cardiomiopatia no puerpério, esteatose maligna da gravidez e pancreatite aguda, representaram um caso cada.

Tabela 1. Causas básicas de óbitos.

\begin{tabular}{ll}
\hline Causa básica de óbito & Número de casos (Percentual) \\
\hline Hipertensivas & 13 casos $(22,81 \%)$ \\
Hemorrágicas & 12 casos $(21,05 \%)$ \\
Infecciosas & 10 casos $(17,54 \%)$ \\
Outros & 10 casos $(17,54 \%)$ \\
Complicação cirúrgica & 4 casos $(7,02 \%)$ \\
Tromboembolismo pulmonar & 4 casos $(7,02 \%)$ \\
Neoplásicas & 3 casos $(5,26 \%)$ \\
\hline
\end{tabular}

O principal período em que ocorreu o óbito foi no puerpério com 41 casos $(71,93 \%)$. O percentual de óbito que ocorreu durante a gestação correspondeu a $8,77 \%$ (5 casos), sendo o mesmo valor correspondente a após abortamento. Óbito durante o abortamento correspondeu a 5,26\% (3 casos), no parto até 1 h a $1,75 \%$ ( 1 caso), e de 43 dias até 1 ano após o parto a $3,51 \%$ (2 casos).

Foram encontrados $66,67 \%$ (30 casos) de preenchimento incorreto na causa básica da morte das declarações de óbito emitidas (total de 45 declarações de óbito). Dentre os motivos para o preenchimento incorreto, consideramos o uso de termos vagos (falência ou disfunção de múltiplos órgãos, parada cardíaca, siglas como AVC, por exemplo) ou uso de termos não classificados na CID-10. O termo falência ou disfunção de múltiplos órgãos foi citado em 22 ocasiões nas DOs analisadas.

$\mathrm{Na}$ análise do preenchimento das declarações de óbito, outros dados relevantes foram: 8,89\% de campos ilegíveis na data do óbito; $40 \%$ de preenchimento incorreto no campo ocupação habitual, dos quais a resposta "do lar ou dona de casa" representou um total de $88,89 \% ; 28,89 \%$ de preenchimento incorreto no campo "o médico que assina atendeu ao falecido?", devido a médicos que assistiram ao óbito marcarem a opção de substituto ou outro. $\mathrm{O}$ não preenchimento de campo correspondeu a 93,33\% no campo "cartão SUS", 26,67\% no campo "naturalidade" e 22,22\% nos campos "estado civil" e "escolaridade". A Tabela 2 traz o resultado absoluto e percentual da análise do preenchimento de cada campo da declaração de óbito.

Tabela 2. Análise do preenchimento da DO.

\begin{tabular}{|c|c|c|c|c|c|}
\hline Campos da DO & Não preenchimento & $\begin{array}{l}\text { Preenchimento } \\
\text { incompleto }\end{array}$ & Campos ilegíveis & $\begin{array}{l}\text { Preenchimento } \\
\text { incorreto }\end{array}$ & $\begin{array}{l}\text { Preenchimento } \\
\text { adequado }\end{array}$ \\
\hline Tipo de óbito & 2 casos $(4,44 \%)$ & 0 casos $(0 \%)$ & 0 casos $(0 \%)$ & 0 casos $(0 \%)$ & $43 \operatorname{casos}(95,56 \%)$ \\
\hline Data do óbito & 0 casos $(0 \%)$ & 0 casos $(0 \%)$ & $4 \operatorname{casos}(8,89 \%)$ & 0 casos $(0 \%)$ & $41 \operatorname{casos}(91,11 \%)$ \\
\hline Cartão SUS & $42 \operatorname{casos}(93,33 \%)$ & 0 casos $(0 \%)$ & 0 casos $(0 \%)$ & 0 casos $(0 \%)$ & $3 \operatorname{casos}(6,67 \%)$ \\
\hline Naturalidade & $12 \operatorname{casos}(26,67 \%)$ & 0 casos $(0 \%)$ & $2 \operatorname{casos}(4,44 \%)$ & 0 casos $(0 \%)$ & 31 casos $(68,89 \%)$ \\
\hline Nome do falecido & 0 casos $(0 \%)$ & 0 casos $(0 \%)$ & 5 casos $(11,11 \%)$ & 1 caso $(2,22 \%)$ & 39 casos $(86,67 \%)$ \\
\hline Nome do pai & 4 casos $(8,89 \%)$ & 0 casos $(0 \%)$ & 4 casos $(8,89 \%)$ & 1 caso $(2,22 \%)$ & 36 casos $(80 \%)$ \\
\hline
\end{tabular}

Continua. 
Conclusão.

Tabela 2. Análise do preenchimento da DO.

\begin{tabular}{|c|c|c|c|c|c|}
\hline Campos da DO & Não preenchimento & $\begin{array}{l}\text { Preenchimento } \\
\text { incompleto }\end{array}$ & Campos ilegíveis & $\begin{array}{l}\text { Preenchimento } \\
\text { incorreto }\end{array}$ & $\begin{array}{l}\text { Preenchimento } \\
\text { adequado }\end{array}$ \\
\hline Nome da mãe & 0 casos $(0 \%)$ & 0 casos $(0 \%)$ & 4 casos $(8,89 \%)$ & 0 casos $(0 \%)$ & $41 \operatorname{casos}(91,11 \%)$ \\
\hline Data de nascimento & 0 casos $(0 \%)$ & 0 casos $(0 \%)$ & 2 casos $(4,44 \%)$ & 0 casos $(0 \%)$ & 43 casos $(95,56 \%)$ \\
\hline Idade & 1 caso $(2,22 \%)$ & 0 casos $(0 \%)$ & 2 casos $(4,44 \%)$ & 0 casos $(0 \%)$ & 42 casos $(93,33 \%)$ \\
\hline Sexo & 1 caso $(2,22 \%)$ & 0 casos $(0 \%)$ & 1 caso $(2,22 \%)$ & 0 casos $(0 \%)$ & 43 casos $(95,56 \%)$ \\
\hline Raça/Cor & 5 casos $(11,11 \%)$ & 0 casos $(0 \%)$ & 0 casos $(0 \%)$ & 0 casos $(0 \%)$ & 40 casos $(88,89 \%)$ \\
\hline Estado civil & 10 casos $(22,22 \%)$ & 0 casos $(0 \%)$ & 0 casos $(0 \%)$ & $0 \operatorname{casos}(0 \%)$ & 35 casos $(77,78 \%)$ \\
\hline Escolaridade & 10 casos $(22,22 \%)$ & $0 \operatorname{casos}(0 \%)$ & 0 casos $(0 \%)$ & 0 casos $(0 \%)$ & 35 casos $(77,78 \%)$ \\
\hline Ocupação habitual & 13 casos $(28,89 \%)$ & 0 casos $(0 \%)$ & 1 caso $(2,22 \%)$ & $18 \operatorname{casos}(40 \%)$ & 13 casos $(28,89 \%)$ \\
\hline Residência & 0 casos $(0 \%)$ & 1 caso $(2,22 \%)$ & 2 casos $(4,44 \%)$ & 1 caso $(2,22 \%)$ & 41 casos $(91,11 \%)$ \\
\hline Local da ocorrência & 1 caso $(2,22 \%)$ & 1 caso $(2,22 \%)$ & 0 casos $(0 \%)$ & $3 \operatorname{casos}(6,67 \%)$ & 40 casos $(88,89 \%)$ \\
\hline $\begin{array}{l}\text { Óbito durante a gravidez, } \\
\text { parto ou aborto }\end{array}$ & $4 \operatorname{casos}(8,89 \%)$ & 0 casos $(0 \%)$ & 0 casos $(0 \%)$ & 1 caso $(2,22 \%)$ & 40 casos $(88,89 \%)$ \\
\hline Óbito durante o puerpério & 4 casos $(8,89 \%)$ & 0 casos $(0 \%)$ & 0 casos $(0 \%)$ & 1 caso $(2,22 \%)$ & 40 casos $(88,89 \%)$ \\
\hline Recebeu assistência médica & $3 \operatorname{casos}(6,67 \%)$ & 0 casos $(0 \%)$ & 0 casos $(0 \%)$ & 1 caso $(2,22 \%)$ & $41 \operatorname{casos}(91,11 \%)$ \\
\hline Necropsia & 5 casos $(11,11 \%)$ & 0 casos $(0 \%)$ & 0 casos $(0 \%)$ & 0 casos $(0 \%)$ & 40 casos $(88,89 \%)$ \\
\hline Causas da morte & 0 casos $(0 \%)$ & $3 \operatorname{casos}(6,67 \%)$ & 0 casos $(0 \%)$ & 30 casos $(66,67 \%)$ & 12 casos $(26,67 \%)$ \\
\hline Nome do médico & 0 casos $(0 \%)$ & 1 caso $(2,22 \%)$ & 0 casos $(0 \%)$ & 1 caso $(2,22 \%)$ & $43 \operatorname{casos}(95,56 \%)$ \\
\hline CRM & 0 casos $(0 \%)$ & 0 casos $(0 \%)$ & 0 casos $(0 \%)$ & 0 casos $(0 \%)$ & $45 \operatorname{casos}(100 \%)$ \\
\hline $\begin{array}{l}\text { O médico que assina atendeu } \\
\text { ao falecido }\end{array}$ & $4 \operatorname{casos}(8,89 \%)$ & 0 casos $(0 \%)$ & 0 casos $(0 \%)$ & 13 casos $(28,89 \%)$ & $28 \operatorname{casos}(62,22 \%)$ \\
\hline Meio de contato & 1 caso $(2,22 \%)$ & 0 casos $(0 \%)$ & 1 caso $(2,22 \%)$ & 0 casos $(0 \%)$ & 43 casos $(95,56 \%)$ \\
\hline Data do atestado & 1 caso $(2,22 \%)$ & 0 casos $(0 \%)$ & 0 casos $(0 \%)$ & 0 casos $(0 \%)$ & $44 \operatorname{casos}(97,78 \%)$ \\
\hline Assinatura & 0 casos $(0 \%)$ & 0 casos $(0 \%)$ & 0 casos $(0 \%)$ & 0 casos $(0 \%)$ & 45 casos $(100 \%)$ \\
\hline
\end{tabular}

A maior parcela das declarações de óbito foram assinadas por médicos plantonistas da Unidade de Terapia Intensiva (UTI) materna (95,56\% das DOs). Duas DOs foram preenchidas por médicos obstetras correspondendo a $4,44 \%$ dos casos.

O ano em que ocorreram mais óbitos foi 2011 com 17 óbitos, seguido de 2012 com 15 óbitos. Apesar da ocorrência de apenas 7 óbitos no ano de 2015 até a data do estudo, houveram 12 óbitos ao total nesse ano. O Gráfico 1 traz a distribuição anual dos óbitos.

Gráfico 1. Distribuição da quantidade de óbitos por ano.

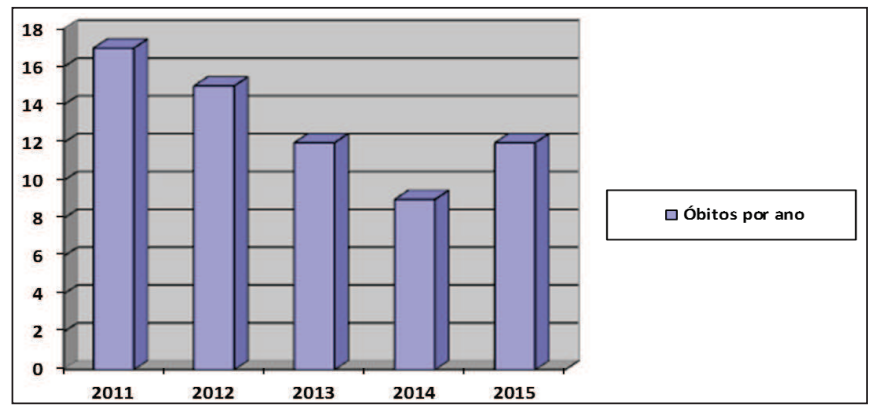

\section{DISCUSSÃO}

O perfil de causas básicas de óbito deste estudo seguiu o mesmo perfil do estado do Ceará e do Brasil: causas obstétricas diretas em primeiro lugar, sendo as principais as causas hipertensivas e hemorrágicas e o principal período o puerpério., $3,6,10$ Já nos Estados Unidos, a partir de 2006, houve redução dos óbitos maternos por causas hemorrágicas e hipertensivas com consequente aumento dos óbitos por doenças cardiovasculares (causa mais comum entre os anos de 2011 a 2013). Em segundo lugar, estão as outras condições médicas que muitas vezes refletem doenças pré-existentes, ${ }^{11}$ o que demonstra a redução de óbito por causas evitáveis, que ainda é a nossa principal realidade.

Apesar do declínio do número de óbitos durante o período do estudo, o Brasil não atingiu o objetivo da Cúpula do Milênio da Organização das Nações Unidas, que era a redução de $75 \%$ das taxas de óbito materno até 2015. Houve apenas redução menor que 50\% entre 1990 e 2015 , o que demonstra a precariedade da nossa assistência à saúde. ${ }^{12}$ 
Sendo a declaração de óbito um registro legal e permanente de grande importância como fonte de informação, pois é através dela que são levantados os dados estatísticos sobre os óbitos e desenvolvidos os programas de saúde, o seu inadequado preenchimento afeta diretamente os dados locais e nacionais sobre mortalidade, e consequentemente as tomadas de decisões dos órgãos públicos. ${ }^{13}$ Dessa forma, são alarmantes os dados encontrados no estudo de preenchimentos incorretos ou não preenchimento dos campos.

A análise do preenchimento das declarações de óbito deste estudo foi feita através das recomendações do manual de instruções para o preenchimento das declarações de óbito elaborado pelo Ministério da Saúde. ${ }^{13}$ Mendonça et al., através de um estudo realizado com médicos de Belo Horizonte sobre o preenchimento das DOs, demonstraram que apenas 50\% dos médicos afirmaram conhecer este manual. Dado alarmante, visto que se trata de um documento de referência essencial para o conhecimento e correto preenchimento das declarações de óbito. ${ }^{14}$

O elevado número de preenchimento incorreto nas causas básicas de óbito avaliado neste estudo $(66,67 \%)$ demonstra a alta deficiência do médico, em sua formação básica, para a elaboração da DO. De forma semelhante, Silva et $a l$, em um estudo conduzido em Belém sobre a qualidade do preenchimento das declarações de óbito, encontraram que $71,5 \%$ das declarações apresentavam algum erro no preenchimento deste campo. O erro mais comum encontrado no estudo de Belém foi a utilização de termos vagos, que estiveram presentes em $440(55 \%)$ das declarações. Os termos vagos mais utilizados em tais estudos, semelhantes ao nosso, foram: falência de múltiplos órgãos e parada cardiorrespiratória. ${ }^{15}$ Da mesma forma, Qaddumi et al., em um estudo conduzido na Palestina, encontraram 92,7\% de termos equivocados utilizados na primeira linha da causa de morte. ${ }^{16}$

\section{REFERÊNCIAS}

1. Brasil. Ministério da Saúde. Secretaria de Atenção à Saúde. Departamento de Ações Programáticas Estratégicas. Manual dos comitês de mortalidade materna. 3. ed. Brasília: Ministério da Saúde; 2009.

2. Say L, Chou D, Gemmill A, Tunçalp Ö, Moller AB, Daniels J, et. al. Global causes of maternal death: a WHO systematic analysis. Lancet Glob Health. 2014;2(6):e323-33.

3. Brasil. Ministério da Saúde. Informações de Saúde: mortalidade, 2010 [Internet]. Brasília: DATASUS; 2011 [acesso em: 05 jun 2015]. Disponível em: http://tabnet.datasus.gov.br/cgi/tabcgi.exe?sim/cnv/ mat10uf.def

4. World Health Organization. The millennium development goals report 2005 [Internet]. Geneva: WHO; 2005 [acesso em: 05 jun 2015]. Disponível em: http://www.who.int/hdp/publications/mdg en.pdf

5. Brasil. Ministério da saúde. OMS: Brasil reduz mortalidade materna em 43\% de 1990 a 2013 [Internet]. Brasília: Ministério da
A percepção de erros graves como uso de siglas e descrição da causa de morte como disfunção de múltiplos órgãos, traz grande preocupação acerca dos dados estatísticos sobre mortalidade materna existentes em nosso meio. Outro preenchimento incorreto comum foi o uso de termos inadequados para descrever a ocupação habitual. Para o correto preenchimento, o termo deve fazer parte da Classificação Brasileira de Ocupações (CBO).

O manual do Ministério da Saúde orienta ainda que "deve ser evitado deixar campos em branco, assinalando a opção "ignorada" quando não se conhecer a informação solicitada ou um traço (-) quando não se aplicar ao item correspondente ou na impossibilidade de serem obtidas as informações". ${ }^{13}$ Apesar da orientação, foram encontrados $93,3 \%$ de não preenchimento do campo "cartão SUS"; $26,6 \%$ de não preenchimento do campo "Naturalidade" e $22,2 \%$ de não preenchimento dos campos "Estado civil e Escolaridade" nesse estudo.

Outro dado relevante foi a constatação de que $100 \%$ das DOs foram assinadas por médicos plantonistas não residentes, ou seja, médicos que não estão mais em treinamento, mas que, de alguma forma, demonstraram necessidade de melhor capacitação para o preenchimento destas.

\section{CONCLUSÃO}

O perfil de mortalidade materna em um hospital escola de referência no estado do Ceará segue o mesmo perfil do Brasil e de países em desenvolvimento.

Devido ao elevado número de preenchimento incorreto das declarações de óbito, sugere-se uma melhor capacitação do profissional médico, tanto na faculdade como através de cursos de atualização profissional para médicos que já concluíram sua formação, uma vez que a DO é um instrumento de preenchimento obrigatório pelo médico, além de principal fonte de informações sobre o perfil de mortalidade do país.

Saúde; 2014 [acesso em: 05 Jun 2015]. Disponível em: http://www. brasil.gov.br/saude/2014/05/oms-brasil-reduz-mortalidadematernaem-43-de-1990-a-2013

6. Governo do Estado do Ceará. Informe epidemiológico mortalidade materna de 09 de junho de 2015 [Internet]. Fortaleza: Secretaria da saúde; 2015 [acesso em: 12 Jun 2015]. Disponível em: http:// www.saude.ce.gov.br/wp-content/uploads/sites/9/2018/06/informe mortalidade_materna_junho_2015.pdf

7. Brasil. Ministério da Saúde. Manual de procedimento do sistema de informações sobre mortalidade. Brasília: Fundação Nacional de Saúde; 2001.

8. Brasil. Ministério da Saúde. Declaração de óbito: documento necessário e importante. Brasília: Ministério da saúde; 2006.

9. Organização Mundial da Saúde. Centro Colaborador da OMS para a Classificação de Doenças em Português. Classificação estatística internacional de doenças em português e problemas relacionados a saúde. 10. Rev. São Paulo: EDUSP; 1994. Volume 2, Manual de Instrução. 
10. Governo do Estado do Ceará. Boletim epidemiológico de mortalidade materna, infantil e fetal de 06 de Abril de 2017 [Internet]. Fortaleza: Secretaria da saúde; 2017.

11. Brown HL, Small MJ. Overview of maternal mortality and morbidity [Internet]. [place unknown]: Uptodate; 2017. [acesso em: 20 Nov 2017]. Disponível em: https://www.uptodate.com/contents/ overview-of-maternal-mortality-and-morbidity

12. Alkema L, Chou D, Hogan D, Zhang S, Moller AB, Gemmill A, et al. Global, regional, and national levels and trends in maternal mortality between 1990 and 2015, with scenario-based projections to 2030: a systematic analysis by the UN Maternal Mortality Estimation Inter-Agency Group. Lancet. 2016;387(10017):462-74.

13. Brasil. Ministério da Saúde. Secretaria de Vigilância em Saúde.
Departamento de Análise de Situação de Saúde. Manual de Instruções para o preenchimento da Declaração de Óbito. Brasília: Ministério da Saúde; 2011.

14. Mendonça FM, Drumond E, Cardoso AM. Problemas no preenchimento da declaração de óbito: estudo exploratório. Rev Bras Estud Popul. 2010;27(2):285-95.

15. Silva JA, Yamaki VN, Oliveira JP, Teixeira RK, Santos FA, Hosoume VS. Declaração de óbito, compromisso no preenchimento: avaliação em Belém - Pará, em 2010. Rev Assoc Med Bras. 2013;59(4):335-40.

16. Qaddumi JA, Nazzal Z, Yacoup AR, Mansour M. Quality of death notifcation forms in North West Bank/Palestine: a descriptive study. BMC Res Notes. 2017;10:154.

\section{Como citar:}

Miná PF, Paiva JP, Feitosa FE, Cordeiro DE. Mortalidade materna e qualidade do preenchimento das declarações de óbito em um hospital escola de referência do Ceará. Rev Med UFC. 2018 out-dez;58(4):40-45. 